\title{
Nonlinear Response of Vibrational Conveyers with Nonideal Vibration Exciter: Superharmonic and Subharmonic Resonance
}

\author{
H. Bayıroğlu, ${ }^{1}$ G. F. Alışverişçi, ${ }^{1}$ and G. Ünal ${ }^{2}$ \\ ${ }^{1}$ Yildiz Technical University, 34349 Istanbul, Turkey \\ ${ }^{2}$ Yeditepe University, 34755 Istanbul, Turkey \\ Correspondence should be addressed to H. Bayıroğlu, hbayir@yildiz.edu.tr
}

Received 17 October 2011; Accepted 24 November 2011

Academic Editor: Swee Cheng Lim

Copyright (C) 2012 H. Bayıroğlu et al. This is an open access article distributed under the Creative Commons Attribution License, which permits unrestricted use, distribution, and reproduction in any medium, provided the original work is properly cited.

\begin{abstract}
Vibrational conveyers with a centrifugal vibration exciter transmit their load based on the jumping method. Common unbalanced-mass driver oscillates the trough. The motion is strictly related to the vibrational parameters. The transition over resonance of a vibratory system, excited by rotating unbalances, is important in terms of the maximum vibrational amplitude produced and the power demand on the drive for the crossover. The mechanical system is driven by the DC motor. In this study, the working ranges of oscillating shaking conveyers with nonideal vibration exciter have been analyzed analytically for superharmonic and subharmonic resonances by the method of multiple scales and numerically. The analytical results obtained in this study agree well with the numerical results.
\end{abstract}

\section{Introduction}

The load-carrying element of a horizontal shaking conveyer performs, as a rule, linear (or sometimes circular or elliptical) symmetrical harmonic oscillations-with a sinusoidal variation of exciting force. In vertical shaking conveyers, the load-carrying element performs double harmonic oscillations: linear along the vertical axis and rotational around that axis (i.e., longitudinal and torsional oscillations). Conveyer drives with centrifugal vibration exciters may have (1) a single unbalanced-mass, (2) two equal unbalancing masses, (3) a pendulum-type unbalanced-mass, (4) four unbalanced-masses in two shafts, (5) four rotating unbalanced-masses for three principal modes of oscillation, that is, linear, elliptical, and circular. To induce strictly oriented linear oscillations of the load-carrying element, the conveyer drive should be arranged so that the line of excitation force passes through the 
inertial centre of the entire oscillating system. Nonideal drives find application in suspended and supported vibrational conveyers and feeders [1].

By the characteristics and adjustment of the elastic support elements (oscillating system), we can distinguish between shaking conveyers with a resonant, subresonant, and superresonant system.

A practical difficulty with unbalanced-mass exciters, observed as early as 1904 by A Sommerfeld, is that local instabilities may occur in operating speed of such devices.

Rocard [2], Mazert [3], Panovko, and I. I. Gubanova [4] have studied the problem of the stability of the unbalanced-mass exciter.

The first detailed study on the nonideal vibrating systems is presented by Kononenko. He obtained satisfactory results by the comparison of the experimental analysis and the approximated method [5].

After this publication, the nonideal problem was presented by Evan-Ivanowski [6] or Nayfeh and Mook [7]. These authors showed that sometimes dynamical coupling between energy sources and structural response that had not to be ignored in reel engineering problems.

Theorical studies and computations of Ganapathy and Parameswaran have indicated the beneficial effect of the "material load" during the starting and transition phase of an unbalanced-mass-driven vibrating conveyor [8].

Bolla et al. analyzed through the multiple scales method a response of a simplified nonideal and nonlinear vibrating system [9].

Götzendorfer in [10] presented a macromechanical model for the transport of granular matter on linear and horizontal conveyors subject to linear, circular or elliptic oscillations and compared it to experimental results [10].

\section{The Governing Equations of the Motion}

The equations of motion for the modified rocard system may be obtained by using Lagrange's equation

$$
\frac{d}{d t}\left(\frac{\partial T}{\partial \dot{q}_{i}}\right)+\frac{\partial D}{\partial \dot{q}_{i}}-\frac{\partial T}{\partial q_{i}}+\frac{\partial V}{\partial q_{i}}=0
$$

where $T$, the kinetic energy, is

$$
T=\frac{1}{2} M \dot{y}^{2}+\frac{1}{2} m\left[(\dot{y}+\dot{\theta} e \cos \theta)^{2}+(\dot{\theta} e \sin \theta)^{2}\right]+\frac{1}{2} I_{\mathrm{mot}} \dot{\theta}^{2},
$$

where $e$ is the eccentricity of the mass $m, m$ is the unbalanced-mass, $M$ is the mass of the trough and the conveyed material on the trough of the conveyor, $\theta$ is the angle of rotation of the shafts carrying unbalanced-masses, $I_{\text {mot }}$ is the moment of inertia of the rotating parts in the motor, $V$, the potential energy, is

$$
V=\frac{1}{2} k_{1} y^{2}+\frac{1}{4} k_{2} y^{4}+m g e(1+\sin \theta)
$$


where the constants $k_{1}$ and $k_{2}$ are the linear and nonlinear elastic coefficients, respectively, $D$, the Rayleigh dissipation function, is

$$
D=\frac{1}{2} c \dot{y}^{2}+\frac{1}{2} K\left(\omega_{s}-\dot{\theta}\right)
$$

and $q_{i}$ is the generalized coordinate. Applying Lagrange's equation for the two coordinates $q_{i}=y$ and $q_{i}=\theta$ gives the differential equations of motion

$$
\begin{gathered}
M \ddot{y}+m \ddot{y}+c \dot{y}+k_{1} y+k_{2} y^{3}=m e\left(\dot{\theta}^{2} \sin \theta-\ddot{\theta} \cos \theta\right) \\
(\underbrace{I_{\mathrm{mot}}+m e^{2}}_{I_{\mathrm{sys}}}) \ddot{\theta}+m e(\ddot{y} \cos \theta+g \cos \theta)=\frac{1}{2}\left[\frac{\partial K}{\partial \dot{\theta}}\left(\dot{\theta}-\omega_{s}\right)+K\right] .
\end{gathered}
$$

Equation (2.5) can be rewritten as follows:

$$
\begin{gathered}
\ddot{y}+\omega_{0}^{2} y=\varepsilon\left[-2 \mu \dot{y}-\alpha y^{3}-e \ddot{\theta} \cos \theta\right]+\kappa\left(\dot{\theta}^{2} \sin \theta\right), \\
\ddot{\theta}=\varepsilon[-I \cos \theta(\ddot{y}+g)+E(\dot{\theta})],
\end{gathered}
$$

where

$$
\begin{gathered}
I=\frac{(m+M) e}{I_{\text {sys }}}, \quad \kappa=\frac{m e}{m+M}, \quad 2 \mu=\frac{c}{m}, \quad \alpha=\frac{k_{2}}{m}, \\
\frac{k_{1}}{m+M}=\omega_{0}^{2}, \quad \frac{m}{m+M}=\varepsilon, \quad E(\dot{\theta})=\frac{(m+M) L(\dot{\theta})}{m I_{\text {sys }}}, \\
L(\dot{\theta})=\frac{1}{2}\left[\frac{\partial K}{\partial \dot{\theta}}\left(\dot{\theta}-\omega_{s}\right)+K\right]
\end{gathered}
$$

where $c$ is the damping coefficient of the vibrating conveyor, $g$ is acceleration due to gravity, $I_{\text {sys }}=\left(I_{m}+I_{M}+I_{\text {mot }}\right)$ is the total moment of inertia of all the rotating parts in the system, and $\omega_{s}$ is the synchronous angular speed of the induction motor [9]. $K$ is the instantaneous drive torque available at the shafts. Note that $E$ contains $L(\dot{\theta})$ that is the active torque generated by the electric circuit of the DC motor, shown in Figure 1.

\section{Analytical Solution}

Ideal system: if there is no coupling between motion of the rotor and vibrating system and $\dot{\theta}=$ constant $(\theta=\omega t, \ddot{\theta}=0),(2.6)$ becomes

$$
\ddot{y}+\omega_{0}^{2} y=\varepsilon\left[-2 \mu \dot{y}-\alpha y^{3}\right]+\kappa \omega^{2} \sin \omega t .
$$

On the right side of the equation, a function of time is present. 


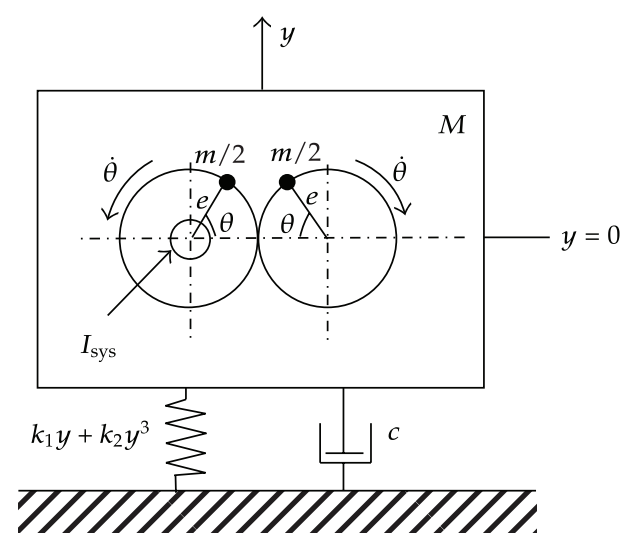

Figure 1: Vibrating model of the system.

Nonideal system:

$$
\begin{gathered}
\ddot{\theta}=\varepsilon[-I \cos \theta(\ddot{y}+g)+E(\dot{\theta})], \\
\ddot{y}+\omega_{n}^{2} y=\varepsilon\left[-2 \mu \dot{y}-\alpha y^{3}-e \ddot{\theta} \cos \theta\right]+\kappa\left(\dot{\theta}^{2} \sin \theta\right),
\end{gathered}
$$

where $E(\dot{\theta})=M_{m}(\dot{\theta})-H(\dot{\theta})$ is the difference between the torque generated by the motor and the resistance torque. Function $E(\dot{\theta})=u_{1}-u_{2} \dot{\theta}$ is approximated by a straight line, where $u_{1}$ is a control parameter that can be changed according to the voltage, and $u_{2}$ is a constant parameter, characteristic for the model of the motor. method:

We will obtain an approximate analytical solution to (3.2) by using the multiple scales

$$
\begin{gathered}
\theta(t, \varepsilon) \approx \theta_{0}\left(T_{0}, T_{1}\right)+\varepsilon \theta_{1}\left(T_{0}, T_{1}\right)+\varepsilon^{2} \theta_{2}\left(T_{0}, T_{1}\right), \\
y(t, \varepsilon) \approx y_{0}\left(T_{0}, T_{1}\right)+\varepsilon y_{1}\left(T_{0}, T_{1}\right)+\varepsilon^{2} y_{2}\left(T_{0}, T_{1}\right), \\
T_{n}=\varepsilon^{n} t, \quad n=0,1, \ldots, \quad T_{0}=t, \quad T_{1}=\varepsilon t
\end{gathered}
$$

where the fast scale $T_{0}=t$ and slow scale $T_{1}=\varepsilon t$. The time derivatives transform according to

$$
\begin{gathered}
\frac{d}{d t}=\frac{d T_{0}}{d t} \frac{\partial}{\partial T_{0}}+\frac{d T_{1}}{d t} \frac{\partial}{\partial T_{1}}+\cdots=D_{0}+\varepsilon D_{1}+\cdots \\
\frac{d^{2}}{d t^{2}}=D_{0}^{2}+2 \varepsilon D_{0} D_{1}+\cdots
\end{gathered}
$$

where $D_{n}=\partial / \partial T_{n},(n=0,1, \ldots)$; then,

$$
\begin{array}{r}
\dot{\theta}=\frac{d \theta}{d t} \approx D_{0} \theta_{0}+\varepsilon\left(D_{1} \theta_{0}+D_{0} \theta_{1}\right)+\cdots, \\
\ddot{\theta}=\frac{d^{2} \theta}{d t^{2}} \approx D_{0}^{2} \theta_{0}+\varepsilon\left(2 D_{0} D_{1} \theta_{0}+D_{0}^{2} \theta_{1}\right)+\cdots,
\end{array}
$$


Mathematical Problems in Engineering

$$
\begin{array}{r}
\dot{y}=\frac{d y}{d t} \approx D_{0} y_{0}+\varepsilon\left(D_{1} y_{0}+D_{0} y_{1}\right)+\cdots, \\
\ddot{y}=\frac{d^{2} y}{d t^{2}} \approx D_{0}^{2} y_{0}+\varepsilon\left(2 D_{0} D_{1} y_{0}+D_{0}^{2} y_{1}\right)+\cdots .
\end{array}
$$

Substituting (3.5) into (3.2), we will obtain

$$
\begin{aligned}
& D_{0}^{2} \theta_{0}=\varepsilon\left[-2 D_{0} D_{1} \theta_{0}-D_{0}^{2} \theta_{1}-I \cos \theta\left(D_{0}^{2} y_{0}+g\right)+E(\dot{\theta})\right] \\
& D_{0}^{2} y_{0}+\varepsilon\left(2 D_{0} D_{1} y_{0}+D_{0}^{2} y_{1}\right)+\omega_{n}^{2}\left(y_{0}+\varepsilon y_{1}\right) \\
& \left.\quad=\varepsilon\left[-2 \mu D_{0} y_{0}-\alpha\left(y_{0}\right)^{3}-e D_{0}^{2} \theta_{0} \cos \theta\right]+\kappa\left[D_{0} \theta_{0}+\varepsilon\left(D_{1} \theta_{0}+D_{0} \theta_{1}\right)\right]^{2} \sin \theta\right]
\end{aligned}
$$

and equating coefficients of a like powers $\varepsilon$, we obtain

(i) for $\varepsilon^{0}$

$$
\begin{gathered}
D_{0}^{2} \theta_{0}=0, \\
D_{0}^{2} y_{0}+\omega_{0}^{2} y_{0}=\kappa\left(D_{0} \theta_{0}\right)^{2} \sin \theta
\end{gathered}
$$

(ii) for $\varepsilon^{1}$

$$
\begin{aligned}
D_{0}^{2} \theta_{1}= & -2 D_{0} D_{1} \theta_{0}-I \cos \theta\left(D_{0}^{2} y_{0}+g\right)+E(\dot{\theta}) \\
D_{0}^{2} y_{1}+\omega_{0}^{2} y_{1}= & -2 D_{0} D_{1} y_{0}-2 \mu D_{0} y_{0}-\alpha\left(y_{0}\right)^{3}-e D_{0}^{2} \theta_{0} \cos \theta \\
& +\kappa 2 D_{0} \theta_{0}\left(D_{1} \theta_{0}+D_{0} \theta_{1}\right) \sin \theta
\end{aligned}
$$

The solution of (3.7) can be written as

$$
\begin{gathered}
\theta_{0}=B T_{0}+\sigma T_{1}, \\
y_{0}=\Lambda \sin \left(T_{0} \Omega\right)+A\left(T_{1}\right) e^{i \omega_{0} T_{0}}+\bar{A}\left(T_{1}\right) e^{-i \omega_{0} T_{0}},
\end{gathered}
$$

where

$$
\begin{gathered}
\cos \left(\theta_{0}+\varepsilon \theta_{1}\right)=\cos \left(\theta_{0}\right)+O(\varepsilon), \quad \sin \left(\theta_{0}+\varepsilon \theta_{1}\right)=\sin \left(\theta_{0}\right)+O(\varepsilon), \\
\Lambda=\frac{-\kappa \Omega^{2}}{\left(\Omega^{2}-\omega_{0}^{2}\right)} .
\end{gathered}
$$


Table 1: Vibrational conveyer parameters in SI units.

\begin{tabular}{cccccccccccccc}
\hline$\varepsilon$ & $\alpha$ & $\mu$ & $c$ & $I$ & $e(m)$ & $E_{1}$ & $E_{0}$ & $g$ & $\omega_{0}$ & $k_{1}$ & $k_{2}$ & $m$ & $M$ \\
\hline 0.05 & 0.01 & 0.01 & 1 & 0.9 & 0.2 & 1.5 & 1.6 & 9.81 & 1 & 2000 & 100 & 5 & 200 \\
\hline
\end{tabular}

\subsection{Subharmonic Resonances $\Omega \approx 3 \omega_{0}$ (Table 1)}

Near resonance:

$$
\begin{gathered}
D_{0} \theta_{0}=\Omega, \\
\Omega=3 \omega_{0}+\varepsilon \sigma .
\end{gathered}
$$

The solution of (3.13) can be written as

$$
\theta_{0}=\Omega T_{0}=3 \omega_{0} T_{0}+\sigma T_{1}
$$

Taking

$$
A\left[T_{1}, T_{2}\right]=\frac{a\left[T_{1}\right]}{2} e^{i \beta\left[T_{1}\right]},
$$

where $a$ and $\beta$ are real, and substituting it into (3.11), we will obtain

$$
\begin{aligned}
y_{0}= & \frac{1}{2} e^{-i T_{0} \omega_{0}-i \beta\left(T_{1}\right)} a\left(T_{1}\right)+\frac{1}{2} e^{i T_{0} \omega_{0}+i \beta\left(T_{1}\right)} a\left(T_{1}\right)+\Lambda \sin \left(T_{0} \Omega\right), \\
\theta_{1}= & \frac{1}{8\left(\Omega^{3}-\Omega \omega_{0}^{2}\right)^{2}} e^{-i\left(T_{0} \omega_{0}+\beta\left(T_{1}\right)\right)} \\
& \times I\left(-4 \Omega^{2} \omega_{0}^{2} a\left(T_{1}\right)\right. \\
& \times\left(\left(1+e^{2 i\left(T_{0} \omega_{0}+\beta\left(T_{1}\right)\right)}\right)\left(\Omega^{2}+\omega_{0}^{2}\right) \cos \left(T_{0} \Omega\right)-2 i\left(-1+e^{2 i\left(T_{0} \omega_{0}+\beta\left(T_{1}\right)\right)}\right) \Omega \omega_{0} \sin \left(T_{0} \Omega\right)\right) \\
& \left.-2 e^{i\left(T_{0} \omega_{0}+\beta\left(T_{1}\right)\right)}\left(\Omega^{2}-\omega_{0}^{2}\right) \cos \left(T_{0} \Omega\right)\left(-4 g+\left(-\kappa \Omega^{2}+\Lambda \omega_{0}^{2}\right) \sin \left(T_{0} \Omega\right)\right)\right) \\
y_{1}=\left(e^{-i\left(3 T_{0} \Omega+4 T_{0} \omega_{0}+3 \beta\left(T_{1}\right)\right)}\right. & \\
& \times\left(-8 e^{3 i \beta\left(T_{1}\right)} \Omega\left(\Omega^{3}+3 \Omega^{2} \omega_{0}-\Omega \omega_{0}^{2}-3 \omega_{0}^{3}\right)\right. \\
& \quad\left(i e^{i T_{0}\left(4 \Omega+3 \omega_{0}\right)} \omega_{0}^{2}\left(3 \alpha \Lambda^{3}+8 i \Lambda \mu \Omega+I \kappa^{2} \Omega^{2}-I \kappa \Lambda \omega_{0}^{2}\right)\left(36 \Omega^{4}-13 \Omega^{2} \omega_{0}^{2}+\omega_{0}^{4}\right)\right. \\
& \quad+i e^{i T_{0}\left(2 \Omega+3 \omega_{0}\right)} \omega_{0}^{2}\left(-3 \alpha \Lambda^{3}+8 i \Lambda \mu \Omega-I \kappa^{2} \Omega^{2}+I \kappa \Lambda \omega_{0}^{2}\right)\left(36 \Omega^{4}-13 \Omega^{2} \omega_{0}^{2}+\omega_{0}^{4}\right) \\
& \quad+4 e^{i T_{0}\left(\Omega+3 \omega_{0}\right)} g I \kappa \omega_{0}^{2}\left(9 \Omega^{4}-10 \Omega^{2} \omega_{0}^{2}+\omega_{0}^{4}\right)+4 e^{i T_{0}\left(5 \Omega+3 \omega_{0}\right)} g I \kappa \omega_{0}^{2}
\end{aligned}
$$


Mathematical Problems in Engineering

$$
\begin{aligned}
& \times\left(9 \Omega^{4}-10 \Omega^{2} \omega_{0}^{2}+\omega_{0}^{4}\right)+8 e^{3 i T_{0}\left(\Omega+\omega_{0}\right)} g I \kappa\left(36 \Omega^{6}-49 \Omega^{4} \omega_{0}^{2}+14 \Omega^{2} \omega_{0}^{4}-\omega_{0}^{6}\right) \\
&-i e^{3 i T_{0}\left(2 \Omega+\omega_{0}\right)} \omega_{0}^{2}\left(4 \Omega^{4}-5 \Omega^{2} \omega_{0}^{2}+\omega_{0}^{4}\right)\left(\alpha \Lambda^{3}+I \kappa\left(\kappa \Omega^{2}-\Lambda \omega_{0}^{2}\right)\right) \\
&\left.-i e^{3 i T_{0} \omega_{0}} \omega_{0}^{2}\left(4 \Omega^{4}-5 \Omega^{2} \omega_{0}^{2}+\omega_{0}^{4}\right)\left(-\alpha \Lambda^{3}+I \kappa\left(-\kappa \Omega^{2}+\Lambda \omega_{0}^{2}\right)\right)\right) \\
&+ 2 e^{2 i \beta\left(T_{1}\right)} \omega_{0}^{2}\left(36 \Omega^{5}+108 \Omega^{4} \omega_{0}^{2}-39 \Omega^{2} \omega_{0}^{3}+\Omega \omega_{0}^{4}+3 \omega_{0}^{5}\right) \\
& \times\left(e^{i T_{0}\left(5 \Omega+2 \omega_{0}\right)}\left(\Omega+\omega_{0}\right)^{2}\left(2 I \kappa \Omega \omega_{0}^{2}+3 \alpha \Lambda^{2}\left(-\Omega+\omega_{0}\right)\right)\right. \\
&+e^{i\left(T_{0}\left(\Omega+4 \omega_{0}\right)+2 \beta\left(T_{1}\right)\right)}\left(\Omega+\omega_{0}\right)^{2}\left(2 I \kappa \Omega \omega_{0}^{2}+3 \alpha \Lambda^{2}\left(-\Omega+\omega_{0}\right)\right) \\
&+e^{i\left(T_{0}\left(\Omega+2 \omega_{0}\right)\right.}\left(\Omega-\omega_{0}\right)^{2}\left(2 I \kappa \Omega \omega_{0}^{2}-3 \alpha \Lambda^{2}\left(\Omega+\omega_{0}\right)\right)+e^{i\left(5 T_{0} \Omega+4 T_{0} \omega_{0}+2 \beta\left(T_{1}\right)\right)} \\
&\left.\times\left(\Omega-\omega_{0}\right)^{2}\left(2 I \kappa \Omega \omega_{0}^{2}-3 \alpha \Lambda^{2}\left(\Omega+\omega_{0}\right)\right)\right) a\left(T_{1}\right)+24 i e^{i\left(T_{0}\left(2 \Omega+\omega_{0}\right)+\beta\left(T_{1}\right)\right)} \alpha \\
& \times \Lambda \Omega \omega_{0}^{2}\left(-\left(-1-2 e^{2 i\left(T_{0} \omega_{0}+\beta\left(T_{1}\right)\right)}+2 e^{2 i\left(T_{0}\left(\Omega+\omega_{0}\right)+\beta\left(T_{1}\right)\right)}+e^{2 i\left(T_{0}\left(\Omega+2 \omega_{0}\right)+2 \beta\left(T_{1}\right)\right)}\right) \Omega\right. \\
&\left.+\left(-1+6 e^{2 i\left(T_{0} \omega_{0}+\beta\left(T_{1}\right)\right)}-6 e^{2 i\left(T_{0}\left(\Omega+\omega_{0}\right)+\beta\left(T_{1}\right)\right)}+e^{2 i\left(T_{0}\left(\Omega+2 \omega_{0}\right)+2 \beta\left(T_{1}\right)\right)}\right) \omega_{0}\right) \\
&\left.\times\left(6 \Omega^{3}+23 \Omega^{2} \omega_{0}+16 \Omega \omega_{0}^{2}+3 \omega_{0}^{3}\right)\right) . \\
& \times\left(36 \Omega^{6}-49 \Omega^{4} \omega_{0}^{2}+14 \Omega^{2} \omega_{0}^{4}-\omega_{0}^{6}\right) a\left(T_{1}\right)^{2}+e^{3 i T_{0} \Omega}\left(1+e^{6 i\left(T_{0} \omega_{0}+\beta\left(T_{1}\right)\right)}\right) \alpha \Omega \\
&\left.\times\left(\Omega^{2}-\omega_{0}^{2}\right)^{2}\left(36 \Omega^{5}+108 \Omega^{4} \omega_{0}-13 \Omega^{3} \omega_{0}^{2}-39 \Omega^{2} \omega_{0}^{2}+\Omega \omega_{0}^{4}+3 \omega_{0}^{5}\right) a\left(T_{1}\right)^{3}\right) \\
&\left.\left(\cos \left(T_{0} \omega_{0}\right)+i \sin \left(T_{0} \omega_{0}\right)\right)\right) /\left(64 \Omega\left(6 \Omega^{2}-5 \Omega \omega_{0}+\omega_{0}^{2}\right)\right. \\
&\left.\Omega_{0}^{3}\right)^{2} \\
& \times(3)
\end{aligned}
$$

Secular terms will be eliminated from the particular solution of (3.8) if we choose $A$ to be a solution of

$$
-2 \varepsilon \sigma^{\prime}+E(\dot{\theta})
$$

In addition to the terms proportional to $e^{\left( \pm i \omega_{0} T_{0}\right)}$ or proportional to $e^{\left[ \pm i\left(\Omega-2 \omega_{0}\right) T_{0}\right]}$ there is another term that produces a secular term in (3.9). We express $\left(\Omega-2 \omega_{0}\right) T_{0}$ in terms of $\omega_{0} T_{0}$ according to

$$
\left(\Omega-2 \omega_{0}\right) T_{0}=\omega_{0} T_{0}+\varepsilon \sigma T_{0}=\omega_{0} T_{0}+\sigma T_{1} .
$$


Table 2: Vibrational conveyer parameters in SI units.

\begin{tabular}{cccccccccccccc}
\hline$\varepsilon$ & $\alpha$ & $\mu$ & $c$ & $I$ & $e(m)$ & $E_{1}$ & $E_{0}$ & $g$ & $\omega_{0}$ & $k_{1}$ & $k_{2}$ & $m$ & $M$ \\
\hline 0.05 & 0.01 & 0.01 & 1 & 0.9 & 0.2 & 1.5 & 1.6 & 9.81 & 10 & 200000 & 100 & 5 & 200 \\
\hline
\end{tabular}

\subsection{Superharmonic Resonances $\Omega \approx(1 / 3) \omega_{0}$ (Table 2)}

We consider

$$
3 \Omega=\omega_{0}+\varepsilon \sigma
$$

In addition to the terms proportional to $e^{\left( \pm i \omega_{0} T_{0}\right)}$ in (3.9), there is another term that produces a secular term in (3.9). This is $-\alpha \Lambda^{3} e^{\left( \pm 3 i \Omega T_{0}\right)} . T_{0}$ eliminate the secular terms, we express $3 \Omega T_{0}$ in terms of $\omega_{0} T_{0}$ according to

$$
3 \Omega T_{0}=\left(\omega_{0}+\varepsilon \sigma\right) T_{0}=\omega_{0} T_{0}+\sigma \varepsilon T_{0}=\omega_{0} T_{0}+\sigma T_{1} .
$$

Eliminating secular terms in equations, taking (3.16), and separating real and imaginary parts, the system of equations solved it is as follows [11]:

$$
\begin{aligned}
y_{0}= & \frac{1}{2} e^{-i T_{0} \omega_{0}-i \beta\left(T_{1}\right)} a\left(T_{1}\right)+\frac{1}{2} e^{i T_{0} \omega_{0}+i \beta\left(T_{1}\right)} a\left(T_{1}\right)+\Lambda \sin \left(T_{0} \Omega\right), \\
\theta_{1}= & \frac{1}{8\left(\Omega^{3}-\Omega \omega_{0}^{2}\right)^{2}} e^{-i\left(T_{0} \omega_{0}+\beta\left(T_{1}\right)\right)} \\
& \times I\left(-4 \Omega^{2} \omega_{0}^{2} a\left(T_{1}\right)\right. \\
& \times\left(\left(1+e^{2 i\left(T_{0} \omega_{0}+\beta\left(T_{1}\right)\right)}\right)\left(\Omega^{2}+\omega_{0}^{2}\right) \cos \left(T_{0} \Omega\right)-2 i\left(-1+e^{2 i\left(T_{0} \omega_{0}+\beta\left(T_{1}\right)\right)}\right) \Omega \omega_{0} \sin \left(T_{0} \Omega\right)\right) \\
& \left.-2 e^{i\left(T_{0} \omega_{0}+\beta\left(T_{1}\right)\right)}\left(\Omega^{2}-\omega_{0}^{2}\right) \cos \left(T_{0} \Omega\right)\left(-4 g+\left(-\kappa \Omega^{2}+\Lambda \omega_{0}^{2}\right) \sin \left(T_{0} \Omega\right)\right)\right), \\
y_{1}=\left(e^{-i\left(2 T_{0} \Omega+5 T_{0} \omega_{0}+3 \beta\left(T_{1}\right)\right)}\right. & \quad\left(-8 e^{3 i \beta\left(T_{1}\right)} \Omega\left(\Omega^{4}-10 \Omega^{2} \omega_{0}^{2}+9 \omega_{0}^{4}\right)\right. \\
& \quad \times\left(4 e^{i T_{0}\left(4 \Omega+5 \omega_{0}\right)} g I \kappa \omega_{0}^{2}\left(\Omega^{2}-\omega_{0}^{2}\right)-4 e^{5 i T_{0} \omega_{0}} g I \kappa \omega_{0}^{2}\left(-\Omega^{2}+\omega_{0}^{2}\right)\right. \\
& \quad+i e^{i T_{0}\left(3 \Omega+5 \omega_{0}\right)} \omega_{0}^{2}\left(4 \Omega^{2}-\omega_{0}^{2}\right)\left(3 \alpha \Lambda^{3}+8 i \Lambda \mu \Omega+I \kappa^{2} \Omega^{2}-I \kappa \Lambda \omega_{0}^{2}\right) \\
& \quad+e^{i T_{0}\left(\Omega+5 \omega_{0}\right)} \omega_{0}^{2}\left(-4 \Omega^{2}+\omega_{0}^{2}\right)\left(3 i \alpha \Lambda^{3}+8 \Lambda \mu \Omega+i I \kappa^{2} \Omega^{2}-i I \kappa \Lambda \omega_{0}^{2}\right) \\
& \left.\quad+8 e^{i T_{0}\left(2 \Omega+5 \omega_{0}\right)} g I \kappa\left(4 \Omega^{4}-5 \Omega^{4} \omega_{0}^{2}+\omega_{0}^{4}\right)\right)+2 e^{2 i \beta\left(T_{1}\right)} \omega_{0}^{2}\left(4 \Omega^{4}-37 \Omega^{2} \omega_{0}^{2}+9 \omega_{0}\right)
\end{aligned}
$$


Mathematical Problems in Engineering

$$
\begin{aligned}
\times & \left(e^{4 i T_{0}\left(\Omega+\omega_{0}\right)}\left(\Omega+\omega_{0}\right)^{2}\left(2 I \kappa \Omega \omega_{0}^{2}+3 \alpha \Lambda^{2}\left(-\Omega+\omega_{0}\right)\right)+e^{2 i\left(3 T_{0} \omega_{0}+\beta\left(T_{1}\right)\right)}\left(\Omega+\omega_{0}\right)^{2}\right. \\
\times & \left(2 I \kappa \Omega \omega_{0}^{2}+3 \alpha \Lambda^{2}\left(-\Omega+\omega_{0}\right)+e^{4 i T_{0} \omega_{0}}\left(\Omega-\omega_{0}\right)^{2}\left(2 I \kappa \Omega \omega_{0}^{2}-3 \alpha \Lambda^{2}\left(\Omega+\omega_{0}\right)\right)\right. \\
& \left.\quad+e^{2 i\left(2 T_{0} \Omega+3 T_{0} \omega_{0}+\beta\left(T_{1}\right)\right)}\left(\Omega-\omega_{0}\right)^{2}\left(2 I \kappa \Omega \omega_{0}^{2}-3 \alpha \Lambda^{2}\left(\Omega+\omega_{0}\right)\right)\right) a\left(T_{1}\right) \\
- & 24 i e^{i \beta\left(T_{1}\right)} \alpha \Lambda \Omega \omega_{0}^{2}\left(4 \Omega^{4}-5 \Omega^{2} \omega_{0}^{2}+\omega_{0}^{4}\right) \\
\times & \left(2 e^{i\left(3 T_{0} \Omega+5 T_{0} \omega_{0}+2 \beta\left(T_{1}\right)\right)}\left(\Omega^{2}-9 \omega_{0}^{2}\right)-2 e^{i\left(T_{0}\left(\Omega+5 \omega_{0}\right)+2 \beta\left(T_{1}\right)\right)}\left(\Omega^{2}-9 \omega_{0}^{2}\right)\right. \\
& -e^{i T_{0}\left(\Omega+3 \omega_{0}\right)}\left(\Omega^{2}-4 \Omega \omega_{0}+3 \omega_{0}^{2}\right)+e^{i\left(3 T_{0} \Omega+7 T_{0} \omega_{0}+4 \beta\left(T_{1}\right)\right)}\left(\Omega^{2}-4 \Omega \omega_{0}+3 \omega_{0}^{2}\right) \\
& +e^{3 i T_{0}\left(\Omega+\omega_{0}\right)}\left(\Omega^{2}+4 \Omega \omega_{0}+3 \omega_{0}^{2}\right)-e^{i\left(T_{0}\left(\Omega+7 \omega_{0}\right)+4 \beta\left(T_{1}\right)\right)} \\
\times & \left.\left(\Omega^{2}+4 \Omega \omega_{0}+3 \omega_{0}^{2}\right)\right) a\left(T_{1}\right)^{2} \\
& +\left(e^{2 i T_{0}\left(\Omega+\omega_{0}\right)}+e^{2 i\left(T_{0}\left(\Omega+4 \omega_{0}\right)+3 \beta\left(T_{1}\right)\right)}\right) \alpha \Omega\left(\Omega^{2}-\omega_{0}^{2}\right)^{2} \\
& \left.\left.\times\left(4 \Omega^{4}-37 \Omega^{2} \omega_{0}^{2}+9 \omega_{0}^{4}\right) a\left(T_{1}\right)^{3}\right)\right) /\left(64\left(-\Omega^{2} \omega_{0}+\omega_{0}^{3}\right)^{2}\left(4 \Omega^{5}-37 \Omega^{3} \omega_{0}^{2}+9 \Omega \omega_{0}^{4}\right)\right) .
\end{aligned}
$$

\section{Stability Analysis}

We analyzed in this system the stability $(a, \gamma)$ in the equilibrium point, using (4.1), where $F$ is the Jacobian matrix of (4.2), and $\gamma_{\text {sub }}=3 \beta\left[T_{1}\right]-T_{1} \sigma\left[T_{1}\right], \gamma_{\text {sup }}=\left[\beta\left[T_{1}\right]-T_{1} \sigma\left[T_{1}\right]\right]$. Stability of the approximate solutions depends on the value of the eigenvalues of the Jacobian matrix $F$ [12]. The solutions are unstable if the real part of the eigenvalues is positives Figures 2(a1) and 2(a2). Figure 2 shows the frequency-response curves for the subharmonic and superharmonic resonance of the unbalanced vibratory conveyor:

$$
\begin{gathered}
a_{\mathrm{sub}}^{\prime}=-\mu a-\frac{3}{8 \omega_{0}} \alpha \Lambda a^{2} \cos [\gamma], \\
\gamma_{\mathrm{sub}}^{\prime}=-\frac{3\left(2 I \kappa \Omega^{2} \omega_{0}^{2}+3 \alpha \Lambda^{2}\left(-\Omega^{2}+\omega_{0}^{2}\right)\right)}{4\left(\Omega^{2}-\omega_{0}^{2}\right) \omega_{0}}+\frac{9}{8 \omega_{0}} \alpha a^{2}-\frac{9}{8 \omega_{0}} \alpha \Lambda a \sin [\gamma]-\sigma, \\
a_{\mathrm{sub}}^{\prime}=-\mu a-\frac{3}{8 \omega_{0}}\left(\alpha \Lambda^{3}+I \kappa\left(\kappa \Omega^{2}-\Lambda \omega_{0}^{2}\right)\right) \cos [\gamma], \\
\gamma_{\mathrm{sub}}^{\prime}=-\frac{\left(2 I \kappa \Omega^{2} \omega_{0}^{2}+3 \alpha \Lambda^{2}\left(-\Omega^{2}+\omega_{0}^{2}\right)\right)}{4\left(\Omega^{2}-\omega_{0}^{2}\right) \omega_{0}}+\frac{3 \alpha a^{2}}{8 \omega_{0}}+\frac{\left(\alpha \Lambda^{3}+I \kappa\left(\kappa \Omega^{2}-\Lambda \omega_{0}^{2}\right)\right) \sin [\gamma]}{8 \omega_{0} a}-\sigma, \\
F=\left\{\partial_{a} f_{1}, \partial_{\gamma} f_{1}\right\},\left\{\partial_{a} f_{2}, \partial_{\gamma} f_{2}\right\}, \quad f_{1}=a^{\prime}, f_{2}=\gamma^{\prime} .
\end{gathered}
$$




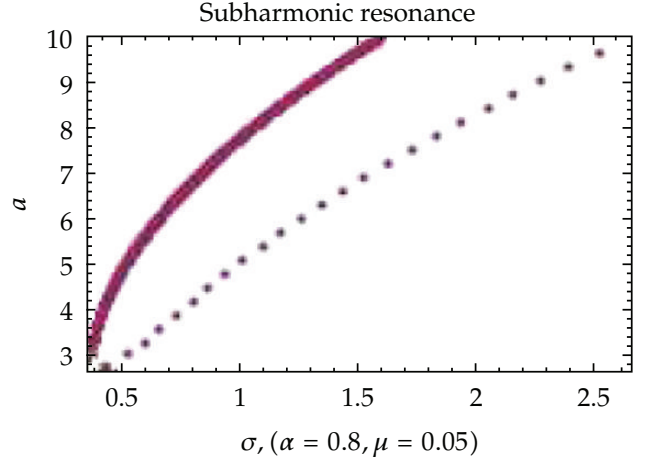

(a1)

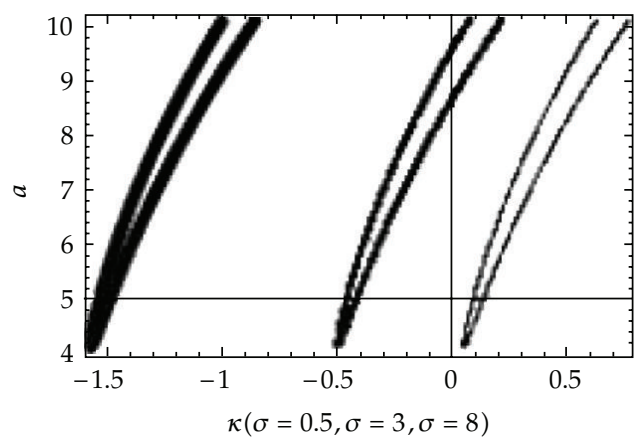

$$
\text { - } \sigma=0.5
$$

1 $\sigma=3$

- $\sigma=8$

(b1)

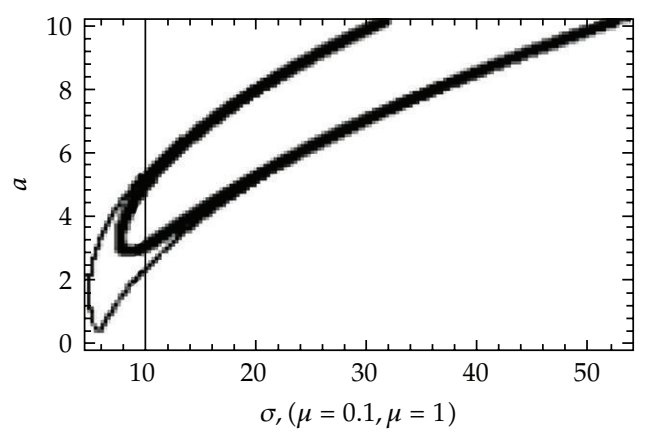

$-\mu=0.1$

- $\mu=1$

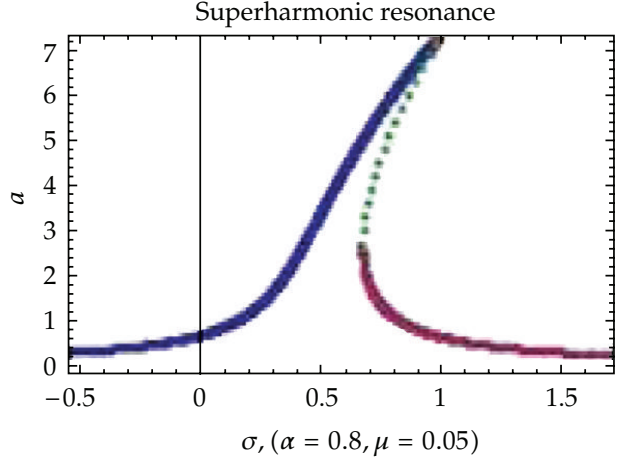

(a2)

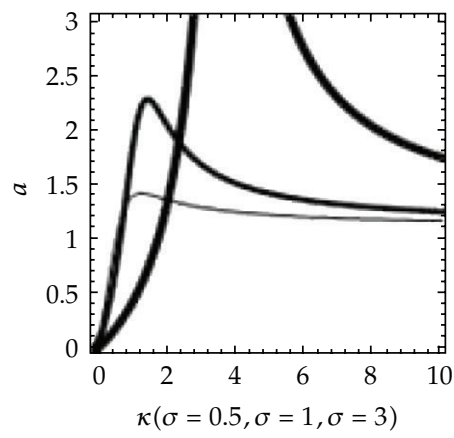

- $\sigma=0.5$

1 $\sigma=1$

- $\sigma=3$

(b2)

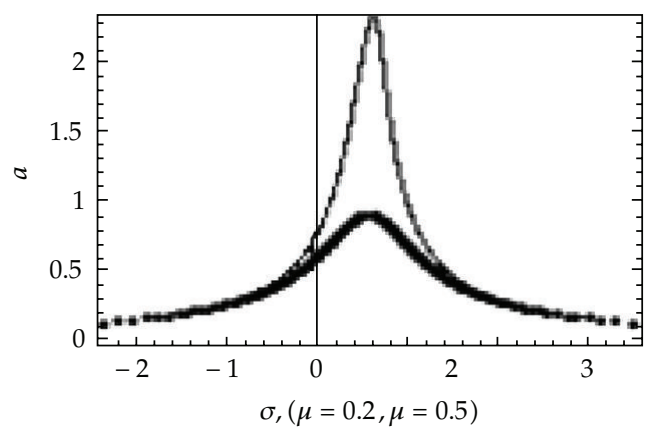

$-\mu=0.2$

- $\mu=0.5$

(c1)

(c2)

Figure 2: Subharmonic resonance and superharmonic resonance. (a) Frequency-response curves with stability, - stable, $\cdots$ unstable $\left\{\left(\mathrm{a}_{1}\right): a=2.78-10, \sigma=0.43258-2.62092\right.$ and $\left(\mathrm{a}_{2}\right): a=2.73-7.21$, $\sigma=0.6782-0.986448\}$, (b) effect of detuning parameter, and (c) effect of damping parameter. 


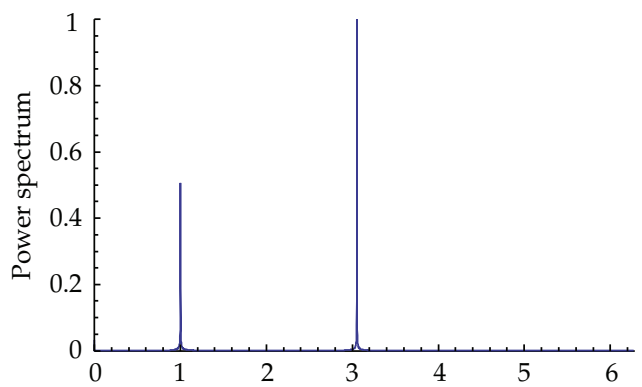

(a)

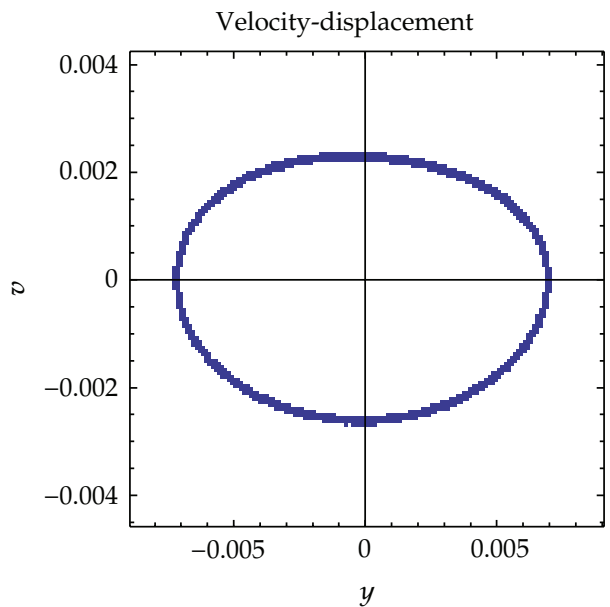

(c)

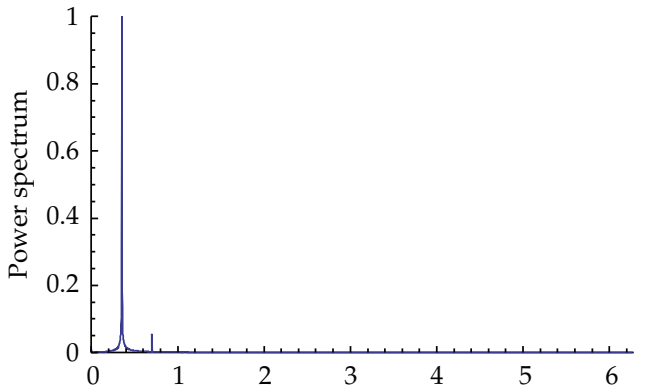

(b)

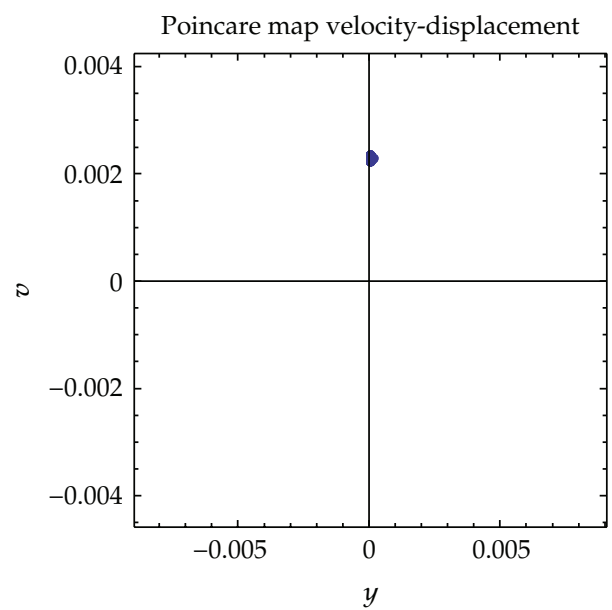

(d)

Figure 3: Subharmonic resonance: (a) power spectrum and superharmonic resonance, (b) power spectrum, (c) phase portrait, and (d) Poincaré sections.

\section{Numerical Results}

The numerical calculations of the vibrating system are performed with the help of the software Mathematica [13, 14]. We analyze the subharmonic resonance $\Omega \approx 3 \omega_{0}$ and superharmonic resonance $3 \Omega \approx \omega_{0}$. Figure 3 shows the power spectrum, phase portrait and the Poincaré map for superharmonic resonance and Figure 3 shows the power spectrum for superharmonic resonance.

\section{Conclusions}

The vibrating system is analyzed, analytically, and numerically for superharmonic and subharmonic resonance by the method of multiple scales. Very often in the motion of the system near resonance the jump phenomenon occurs. The frequency-response curves of the subharmonic resonance consist of two branches; the left one is stable and the right one is unstable (saddle node bifurcation). The frequency-response curves of the superharmonic resonance consist of three branches; the left one is stable, the middle one is unstable, and the right one is stable (pitchfork bifurcation). The stable motions of the oscillator are shown 
with one peaks in the power spectrum for superharmonic resonance and with two peaks in the power spectrum for subharmonic resonance. Both analytical and numerical results that we have obtained are in good agreement. The system studied here exhibits chaotic behaviour in case of strong nonlinearity. This will be reported in the forthcoming paper. Furthermore, control methods in the passage through resonance await for future publication.

\section{Acknowledgment}

The authors greatly appreciate the comments of an anonymous referee.

\section{References}

[1] J. M. Balthazar, D. T. Mook, H. I. Weber et al., "An overview on non-ideal vibrations," Mechanica, vol. 38, no. 6, pp. 613-621, 2003.

[2] Y. Rocard, General Dynamics of Vibrations, Ungar, New York, NY, USA, 3rd edition, 1960.

[3] R. Mazert, Mécanique Vibratoire, C. Béranger, editor, Paris, France, 1955.

[4] Y. G. Panovko and I. I. Gubanova, Stability and Oscillations of Elastic Systems, Consultans Bureau, New York, NY, USA, 1965.

[5] V. O. Kononenko, Vibrating Problems with a Limited Power Supply, Iliffe, London, UK, 1969.

[6] R. M. Evan-Ivanowski, Resonance Oscillations in Mechanical Systems, Elsevier, Amsterdam, The Netherlands, 1976.

[7] A. H. Nayfeh and D. T. Mook, Nonlinear Oscillations, John Wiley \& Sons, New York, NY, USA, 1979.

[8] S. Ganapathy and M. A. Parameswaran, "Transition over resonance and power requirements of an unbalanced mass-driven vibratory system," Mechanism and Machine Theory, vol. 21, no. 1, pp. 73-85, 1986.

[9] M. R. Bolla, J. M. Balthazar, J. L. P. Felix, and D. T. Mook, “On an approximate analytical solution to a nonlinear vibrating problem, excited by a nonideal motor," Nonlinear Dynamics, vol. 50, no. 4, pp. 841-847, 2007.

[10] A. Götzendorfer, Vibrated granular matter: transport, fluidization, and patterns, Ph.D. thesis, University Bayreuth, 2007.

[11] V. Piccirillo, J. M. Balthazar, and B. R. Pontes Jr., "Analytical study of the nonlinear behavior of a shape memory oscillator: part II-resonance secondary," Nonlinear Dynamics, vol. 60, no. 4, pp. 513-524, 2010.

[12] J. Awrejcewicz and C. H. Lamarque, Bifurcation and Chaos in Nonsmooth Mechanical Systems, World Scientific, River Edge, NJ, USA, 2003.

[13] A. H. Nayfeh and C. M. Chin, Perturbation Methods with Mathematica, Dynamics Press, Virgina, va, USA, 1999.

[14] S. Lynch, Dynamical Systems with Applications Using Mathematica, Birkhäauser, Boston, Mass, USA, 2007. 


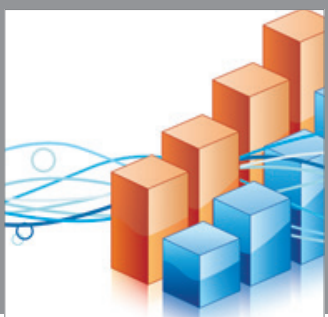

Advances in

Operations Research

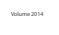

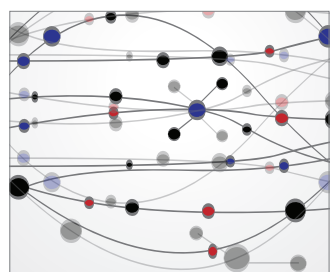

\section{The Scientific} World Journal
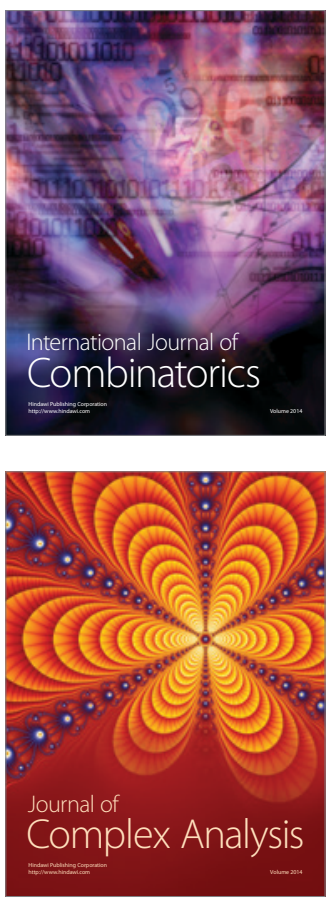

International Journal of

Mathematics and

Mathematical

Sciences
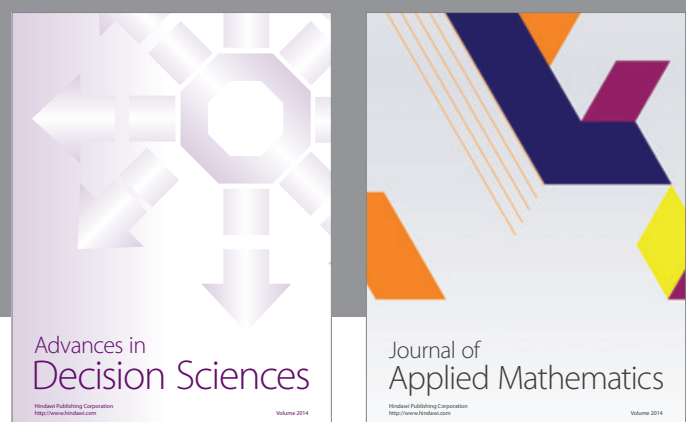

Journal of

Applied Mathematics
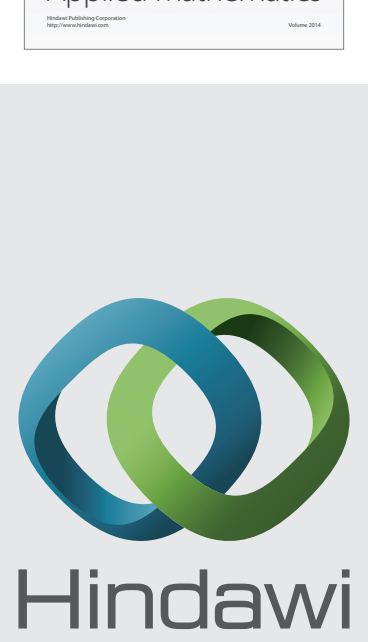

Submit your manuscripts at http://www.hindawi.com
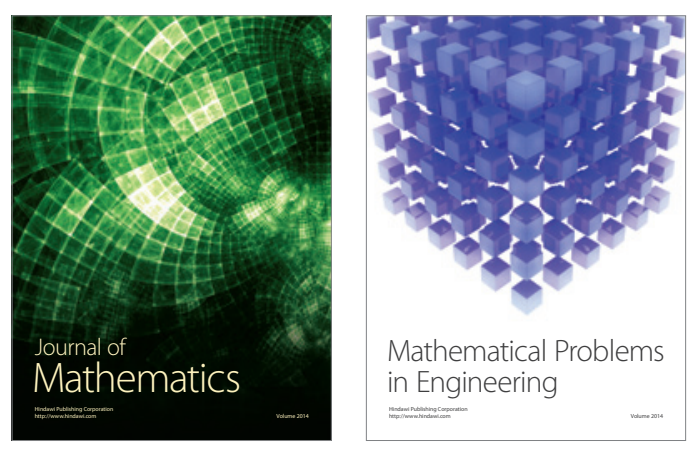

Mathematical Problems in Engineering
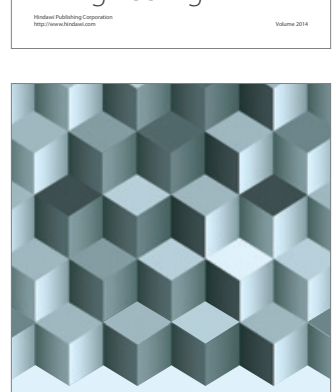

Journal of

Function Spaces
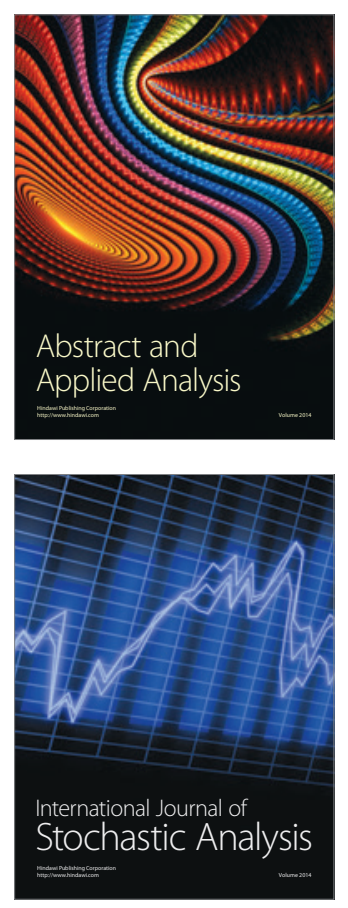

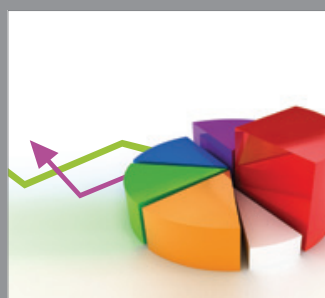

ournal of

Probability and Statistics

Promensencen
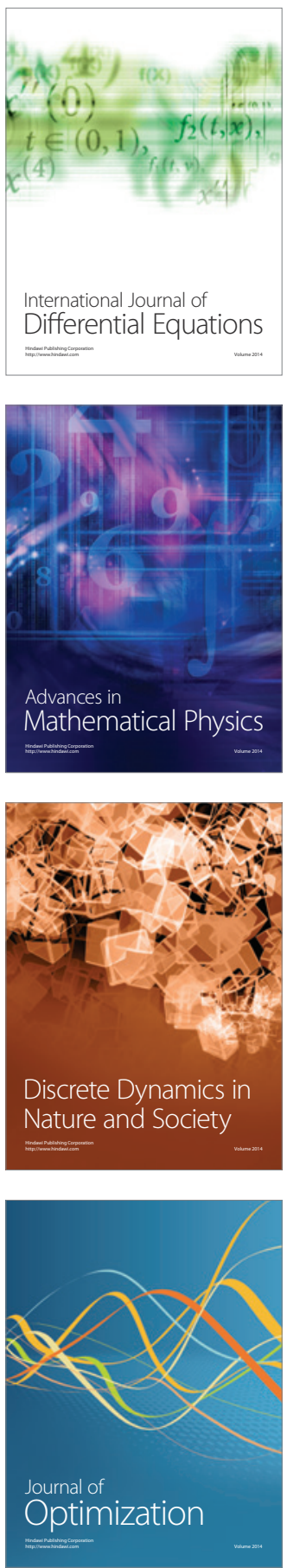